\title{
Winke für die Benutzung des Buches.
}

Die Darstellung der seltenen Erden macht bei dem präparativen Arbeiten mit anorganischen Körpern eine Ausnahme, denn es gelingt nicht durch eine oder wenige Operationen irgend eine Erde rein zu gewinnen. Da die Anzahl der seltenen Erden eine verhältnismäBig große ist und $\mathrm{ihr}$ Verhalten infolge der nahen verwandtschaftlichen Beziehungen sich sehr ähnelt, ist man darauf angewiesen, Kristallisationen oder Fällungen fraktioniert vorzunehmen and erreicht hierdurch nur auf sehr mühevollem und langwierigem Wege das erwünschte Ziel.

Eine Trennungsmethode erlaubt fast nie die Reindarstellung einer Erde, man muB vielmehr zur Kombination mehrerer Methoden greifen, und die Wahl der Methoden sowie das Beginnen mit einem neuen Verfahren richtet sich ausschlieBlich nach dem Reinheitsgrad der Fraktionierungsgrenzen.

So kommt es, daB man von vornherein fast nie fur die Darstellung einer Erde positive Direktiven geben kann; dieselben wird man sich für jeden einzelnen Fall selbst aufstellen.

Aus diesen wenigen Andeutungen geht schon zur Genüge hervor, daB die Anordnung des Stoffes in diesem Buche eine ihm eigentümliche sein muB. An der Hand eines Beispieles dürte der Gebrauch des Buches sich am leichtesten erklären lassen. Gesetzt den Fall, es wird jemand vor die Aufgabe gestellt, Lanthanerde darzustellen und will als Wegweiser und Auskunftsmittel sich dieses Buches bedienen.

Der erste Abschnitt soll einen allgemeinen Überblick über die Geschichte der Entdeckung der seltenen Erden gewähren, 
der zweite den Leser mit den charakteristischen Merkmalen der verschiedenen Erdgruppen und einzelnen Erden vertraut machen.

Es handelt sich sodann um das für diese Zwecke in Betracht kommende Ausgangsmaterial. Im Abschnitt „Wahl des Ausgangsmaterials" (Bd. II, S. 128) finden sich für jede Erde mehrere Mineralien oder Nebenprodukte der Thoriumfabrikation usw. angeführt. Angenommen, man entscheidet sich aus bestimmten Gründen für das Mineral Cerit. Die nächste Frage, welche Erden enthält der Cerit und wie schlieBe ich ihn auf, beantwortet der Abschnitt „AnfschlieBen der wichtigsten Mineralien als Ausgangsmaterial“ (Bd. II, S. 73). Nachdem das Mineral aufgeschlossen ist, hat man sich für die Abscheidungsart zu entschliessen und findet im Abschnitt (Bd. II, S. 46) „Fällen der seltenen Erden" die erforderlichen Hinweise.

Sind auf diese Weise die Roherden in Form von Oxalaten hergestellt, so muB man sich über die zu wählenden Trennungsmethoden schlüssig werden.

Aus den Analysen des Cerits (Bd. II, S. 73) ersieht man, daB es sich fast ausschlieBlich um Ceriterden handelt, denn die geringen Mengen Yttererden können vorläufig unberücksichtigt bleiben, da sie durch die später vorzunehmenden Manipulationen in Endfraktionen im angereicherten Zustand erscheinen.

Der letzte Abschnitt des ersten Bandes enthält eine $\mathrm{Zu}$ sammenstellung der gebräuchlichen Methoden, die für die Darstellung der einzelnen Erden in Betracht kommen. Unter „Lanthan" sind sämtliche Methoden angeführt, die für die Darstellung dieser Erde benutzt wurden. Nach den bisherigen Erfahrungen eignen sich die Ammondoppelnitrate für den genannten Zweck am besten, so daB man sich auch für sie entscheiden wird.

Die Trennungsmethode mittels der Ammondoppelnitrate (Bd. I, S. 418) wird nach entsprechender Einsicht alle Anhaltspunkte für eine Direktive bieten, so daB man zu der sofortigen Überführung der Oxalate in die Doppelsalze schreitet. 
Da zur Abscheidung des Cers diese Erde in der vierwertigen Form vorliegen muB, so wird man sich für die $0 x y$ dation auf trocknem oder nassem Wege zu entscheiden haben und hierüber Auskunft im Abschnitt „Ü̉berführen der Oxalate in Nitrate, Sulfate, Oxyde usw.“ (Bd. II, S. 58) holen.

Nachdem die Hauptmenge des Cers in Form von Ceriammonnitrat abgeschieden ist, kann der Rest unberücksichtigt bleiben und die Ammondoppelnitrate auf Lanthan fraktioniert kristallisiert werden. Die Beschreibung dieser Trennungsmethode bringt alle Angaben, die für ein schnelles Gelingen erforderlich sind. Liegt die Lanthanerde schon in reinerem Zustande vor und will man die sie begleitende Cererde gänzlich entfernen, so findet man im fünften Abschnitt unter Cer die in Betracht kommenden Methoden angeführt, jedoch enthält das Kristallisationsverfahren der Ammondoppelnitrate genügende Hinweise, so daß man nie fehlgehen kann.

Um aber auf kürzerem Wege eine Anreicherung des Lanthans zu erhalten, kann man sich anderer Methoden bedienen, wie z. B. der partiellen Löslichkeit der Oxalate in Mineralsäuren oder mehrerer basischen Methoden. Alle diesbezüglichen Fingerzeige sind, wie schon oben erwähnt, im SchluBkapitel des ersten Bandes berücksichtigt.

Den Fortgang des Fraktionierens kontrolliert man durch die Spektralanalyse ${ }^{1}$, die im zweiten Bande (S. 291) beschrieben ist.

Die zur Feststellung des endgultigen Reinheitsgrades nötige Auskunft bringen die Kapitel „Atomgewichtsbestimmungsmethoden“ (Bd. II S. 187) und Atomgewichte (Bd. II S. 214) der seltenen Erdmetalle.

Sollte es sich um event. Verunreinigungen anderer Metalle handeln, die durch die Reagentien oder GefäBe in das Präparat hineingelangt sind, so informiert man sich im Kapitel „Trennung

1 Handelt es sich um Elemente farbloser Erden, und besonders um solche mit sehr abweichenden Atomgewichten, so wird man schneller und zuverlässiger Unterschiede in der Zussmmensetzung von Erdgemischen durch Bestimmung des Äquivalentgewichtes (Bd. II S. 187) konstatieren. 
der seltenen Erden von anderen Metallen" (Bd. II, S. 1). Während nach den Angaben dieses Kapitels (s. auch Bd. II, S. 134) eine vollständige qualitative Analyse ausgefuhrt werden kann, sind die für die quantitative Bestimmung des Cers, Thoriums und Praseodyms erforderlichen Methoden in dem darauf folgenden Kapitel (Bd. II, S. 138) beschrieben.

Weitere Direktiven für den Gebrauch des Buches zu geben, dürfte überflüssig sein, da aus der Inhaltsübersicht die Anordnung des Stoffes hervorgeht und das ausführliche Register über jedes Stichwort Auskunft erteilt. 